\title{
LA ADQUISICIÓN DE LA ASIMILACIÓN DE SONORIDAD
}

\author{
ZSUZSANNA BÁRKÁNYI \\ Universidad Eötvös Loránd de Budapest
}

\begin{abstract}
In the present article we will display the results of a pilot study on the acquisition of the Spanish laryngeal system by advanced L2 learners. The study contributes to our knowledge about phonological transfer between L1 and L2, in this particular case the acquisition of the voicing properties of Spanish and voicing assimilation in Spanish by native speakers of Hungarian.

En el presente trabajo pretendemos presentar los resultados de un estudio piloto sobre la adquisición del sistema laríngeo de L2 (segunda lengua/lengua extranjera) por parte de alumnos de nivel avanzado. Nuestro objetivo es hacer una contribución a los conocimientos sobre los mecanismos de transferencia de características fonológicas entre la L1 (lengua materna) y la L2. En este caso particular, se analizará la adquisición de las características de sonoridad y la asimilación de sonoridad en el español por parte de los alumnos universitarios húngaros que estudian filología hispánica. Primero presentaremos el contexto más amplio del fenómeno, luego pasaremos a los detalles metodológicos y los resultados de nuestro experimento piloto y al final hablaremos sobre las posibles líneas de investigación en el futuro.
\end{abstract}

1. Los antecedentes: estudios sobre la adquisición de las características laríngeas en $\mathrm{L} 2$

Las lenguas del mundo difieren en cuanto al contraste laringal de las obstruyentes que poseen. En un estudio ya clásico, Lisker y Abramson (1964) ${ }^{1}$ clasificaron las oclusivas desde el punto de vista del periodo de sordez entre la explosión de la oclusiva y el momento de inicio de la sonoridad, a lo que llamaron Voice Onset Time (conocido en la literatura sobre el tema como VOT). Lisker y Abramson hablan de tres grupos de lenguas: al primero pertenecen las que poseen un contraste laríngeo binario como el castellano, el húngaro o el inglés; en el segundo las oclusivas contrastan en tres niveles (p. ej. el coreano, el tailandés, etc.) y al último pertenece el hindi o maratí que poseen un contraste de cuatro niveles. Solamente el primer grupo es de interés para el presente trabajo

${ }^{1}$ Leigh LISKER y Arthur S. ABRAMSON, "A cross-language study of voicing in initial stops: Acoustical measurements", Word, 20, 1964, 384-422. 
puesto que es al que pertenecen tanto el español como el húngaro. Sin embargo, en este grupo también hay diferencias considerables, ya que en inglés el contraste se basa en el VOT corto en las oclusivas fonológicamente sonoras y en el VOT largo (i.e. aspiración) en la pareja sorda, mientras que en castellano la oclusiva sonora se realiza con un VOT negativo, es decir, la vibración de las cuerdas vocales empieza antes de la explosión, y la pareja sorda se realiza con un VOT corto que puede llegar a ser cero. El húngaro es idéntico al español en cuanto al VOT, lo que significa que la codificación del contraste de sonoridad es idéntica en las dos lenguas y, por lo tanto, no esperamos ni encontramos ninguna interferencia en este aspecto. Naturalmente, la adquisición del sistema laringal de una segunda lengua no consiste solamente en la adquisición de un contraste nuevo -sea en sentido fonético, articulatorio o en sentido fonológicosino también en la adquisición de los procesos que alteran la sonoridad de los segmentos.

No hay una abundancia de estudios sobre la adquisición en L2 de las reglas de asimilación y la mayoria de ellos se ocupa del inglés como L $2{ }^{2}$. Como acabamos de ver el castellano y el húngaro codifican el contraste de sonoridad de la misma manera. A pesar de esta similitud que incluye nuestras lenguas en el mismo grupo tipológico, quisiéramos destacar algunas diferencias en los procesos de sonoridad entre las dos lenguas. A fin de poder entender estos procesos mejor, ahora pasamos a la descripción de la sonoridad en los dos idiomas.

\section{El contraste de sonoridad en el español y en el húngaro}

Empezaremos con el sistema húngaro que es un sistema simétrico ya que todas las obstruyentes forman parte de pares de sorda-sonora y los procesos fonológicos relacionados con la sonoridad parecen ser más "regulares". En el Cuadro $1^{3}$ se pueden ver las obstruyentes húngaras.

\footnotetext{
${ }^{2}$ Se recomienda que el lector interesado vea Ellen SIMON, "Phonological Transfer of Voicing and Devoicing Rules. Evidence from L1 Dutch and L2 English Conversational Speech", Language Sciences 32, 2010, 63-86.

3 El cuadro no contiene el segmento glotal [h] que normalmente también se clasifica como obstruyente (véase Péter, SIPTÁR, A mássalhangzók, in: Ferenc, KIEFER (ed.) Strukturális magyar nyelitan 2. Fonológia, Budapest, Akadémiai kiadó, 1994) y no tiene pareja sonora. Su participación en la asimilación de sonoridad también es asimétrica. En este trabajo no trataremos este fonema más detalladamente.
} 


\begin{tabular}{|l|c|c|c|c|c|c|c|c|c|c|}
\hline & \multicolumn{2}{|c|}{ labiales } & \multicolumn{2}{c|}{ dentales/alveolares } & \multicolumn{2}{c|}{ alveo-palatales } & \multicolumn{2}{c|}{ palatales } & \multicolumn{2}{c|}{ velares } \\
\hline & sonora & sorda & sonora & sorda & sonora & sorda & sonora & sorda & sonora & sorda \\
\hline oclusivas & $\mathrm{b}$ & $\mathrm{p}$ & $\mathrm{d}$ & $\mathrm{t}$ & & & $\mathrm{J}$ & $\mathrm{c}$ & $\mathrm{g}$ & $\mathrm{k}$ \\
\hline fricativas & $\mathbf{v}$ & $\mathrm{f}$ & $\mathbf{z}$ & $\mathrm{s}$ & 3 & $\mathrm{~s}$ & & & & \\
\hline africadas & & & $\mathrm{d} z$ & $\mathrm{t}$ & $\mathrm{b}$ & $\mathrm{t}$ & & & & \\
\hline
\end{tabular}

Cuadro 1: Las obstriyentes búngaras

En el húngaro, a diferencia de las lenguas eslavas o el alemán, las obstruyentes finales mantienen el contraste laringeo, es decir, a pesar de la dificultad aerodinámica de mantener la vibración de las cuerdas vocales en posición final, el contraste se mantiene con la ayuda de claves secundarias como la longitud de la consonante y/o la de la vocal anterior.

En cuanto a la asimilación de sonoridad dejaremos aparte el comportamiento asimétrico de la /v/ - es un proceso regresivo (se aplica de derecha a izquierda), se da tanto a través de frontera morfológica dentro de la palabra, p. ej. més $z^{+b o ́ l}$ [zb] 'cal elativo', como en la frontera entre dos palabras kész borostyán [zb] 'hiedra hecha', - es iterativo: barack+böl [dzgb] 'melocotón elativo'. El fenómeno se observa estáticamente también, es decir, monomorfémicamente las obstruyentes son idénticas en cuanto a su sonoridad, no hay palabras húngaras que tengan obstruyentes adyacentes que no concuerden en sonoridad (labda 'pelota', pero *[pd]; táska 'bolso' pero *[3k]). Las resonantes no participan en la asimilación: tréfa 'chiste', draga 'caro'.

En lo que se refiere al sistema consonántico del castellano, es a la vez más simple y más complejo. Como vemos en el Cuadro 2, hay menos obstruyentes, sin embargo, su realización varía más.

\begin{tabular}{|l|c|c|c|c|c|c|c|c|c|c|}
\hline & \multicolumn{2}{|c|}{ labiales } & \multicolumn{2}{c|}{ dentales/alveolares } & \multicolumn{2}{c|}{ alveo-palatales } & \multicolumn{2}{c|}{ palatales } & \multicolumn{2}{c|}{ velares } \\
\hline & sonora & sorda & sonora & sorda & sonora & sorda & sonora & sorda & sonora & sorda \\
\hline oclusivas & $\mathrm{b}$ & $\mathrm{p}$ & $\mathrm{d}$ & $\mathrm{t}$ & & & & & $\mathrm{g}$ & $\mathrm{k}$ \\
\hline fricativas & & $\mathrm{f}$ & & $\theta / \mathrm{s}$ & & & $\mathrm{j}$ & & & $\mathrm{x}$ \\
\hline africadas & & & & & & $\mathrm{tf}$ & & & & \\
\hline
\end{tabular}

Cuadro 2: Las obstruyentes españolas

4 Véase Zsuzsanna BÁRKÁNYI y Zoltán KISS, "On the border of phonetics and phonology: Sonorant voicing in Hungarian and Slovak", Presentación en 20th Manchester Phonology Meeting, 2012, y Zsuzsanna BÁRKÁNYI y Katalin MÁDY, "The perception of voicing in fricatives", Presentación en 9th Old World Conference in Phonology, 2012. 
Es evidente a primera vista que las obstruyentes no forman parejas de sordasonora como en húngaro, excepto las oclusivas ${ }^{5}$. Esto corresponde a tendencias generales puesto que para mantener la turbulencia (fricción) y la vibración de las cuerdas vocales se necesitan gestos articulatorios contradictorios, lo que causa un balance frágil. La turbulencia se produce si el aire fluye con suficiente velocidad, lo que se puede conseguir con la glotis abierta y una hendidura estrecha en la cavidad oral. Esto también significa que la presión del aire debajo de la glotis es tan grande o menor que dentro de la cavidad bucal. Al contrario, para mantener la vibración laríngea las cuerdas vocales tienen que cerrarse suavemente. La presión del aire crece debajo de la glotis, abre las cuerdas vocales, por lo que decrece la presión del aire debajo de la glotis y las cuerdas vocales se cierran otra vez. Si la presión del aire encima de la glotis excede a la de debajo, la vibración cesa y la sonoridad desaparece. ${ }^{6}$ Esto explica por qué hay lenguas que tienen fricativas sordas (p. ej. coreano) pero no tienen sonoras y no al revés. En substancia, el español también es una lengua que omite algunas realizaciones del segmento $/ \mathrm{d} /$. Esto ya nos hace suponer que el castellano será una lengua con neutralización de sonoridad en posición final de palabra a diferencia del húngaro. No obstante, tenemos muy poco terreno para averiguarlo porque las reglas fonotácticas del español son muy estrictas y las únicas obstruyentes que ocurren en posición final de palabra en el patrimonio léxico son la /d/, que en el estándar peninsular se neutraliza con la $/ \theta /$, la $/ \mathrm{s} /$ y en muy pocas palabras la $/ \mathrm{x} /$. En los pocos préstamos que contienen una obstruyente sonora como $c l u b$ o pub, si el segmento final se realiza, normalmente es sorda y sin explosión.

¿Qué ocurre con las obstruyentes en coda dentro de la palabra? Gran parte $(68,86 \%)$ de las sílabas españolas son abiertas. ${ }^{7}$ La mayoría de las codas están formadas por una consonante resonante o / $/$. Ahora dejamos aparte la aspiración de las codas implosivas que hasta cierto grado caracteriza todas las variedades del mundo hispanohablante. Las oclusivas en posición pos-nuclear forman muy pocas oposiciones, por ejemplo: acto-apto, absorción-adsorción y sus realizaciones abarcan una gama muy amplia. Se considera normativo para la secuencia $/ \mathrm{kt} /[\mathrm{kt}],[\mathrm{gt}],[\gamma \mathrm{t}]$, pero también encontramos $[\theta \mathrm{t}]$, $[\mathrm{xt}]$, [st] e incluso la vocalización de la consonante implosiva. En el caso en el que las consonantes difieren en su sonoridad como en absorción, la situación es muy parecida: la

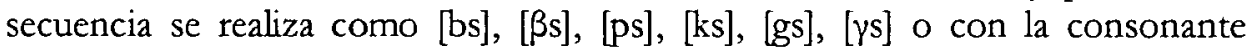

\footnotetext{
${ }^{5}$ En este trabajo no trataremos la variación alofónica de las oclusivas sonoras.

"John J. OHALA, "The origin of sound pattems in the vocal tract constraints", in: P. F. MacNeilage (ed.), The production of speech, New York, Springer-Verlag, 1983, $189-216$.

7 Antonio QUILIS, Tratado de fonética y fonologia españolas, Madrid, Gredos, Biblioteca Románica, 1993.
} 
vocalizada. ${ }^{8}$ Lo que es obvio a primera vista es que la asimilación de sonoridad es opcional, no es tan general como en húngaro. Los estudios sobre la asimilación de sonoridad en castellano son escasos y en su mayoría tratan la sibilante alveolar /s/, así que no hemos encontrado estudios acústicos sobre enunciados como David viene, pero nuestra opinión es que en esta frase la /d/ se puede realizar tanto sorda como sonora y en la mayoría de los casos la /b/ se realiza como una aproximante o espirante $[\beta]$, por lo tanto no proporciona un contexto para la asimilación regresiva entre obstruyentes.

La sonorización de la / $/$ / ha suscitado mucho más interés entre la comunidad internacional de fonólogos. ${ }^{9}$ La sonorización de la /s/ delante de una consonante sonora ocurre en todos los dialectos (['razgo] vs. [rasko]), normalmente se considera gradual, variable y dependiente del estilo. Aquí cabe subrayar que en el dialecto peninsular normativo la sonorización de la /s/ ocurre no solamente delante de obstruyentes sonoras, sino delante de resonantes también ([mizmo]) aunque no en posición pre-vocálica ([pre'sente]). Teniendo en cuenta la fonotáctica del español y la tendencia universal según la cual las fricativas se sonorizan con más facilidad en contextos coarticulatorios que las obstruyentes y que entre las fricativas es la /s/ la que tiende más a sonorizarse $^{10}$, la asimilación de la sibilante española no es sorprendente. Como vemos, esto es una diferencia significativa entre el húngaro y el castellano. En pocas palabras, en húngaro las resonantes no participan en la asimilación de sonoridad y el proceso es categórico, mientras en castellano el proceso es gradual y variable y las resonantes también asimilan la sibilante anterior. Cabe destacar que es llamativo el hecho de que las resonantes sonoricen pero que la posición intervocálica no provoque la sonorización de las obstruyentes sordas. Antes de ver hasta qué grado aprenden estas diferencias subfonémicas los alumnos húngaros de nivel avanzado, discutiremos las dificultades y puntos de interés en la recopilación de datos.

\footnotetext{
${ }^{8}$ QUILIS, op. cit.

${ }^{9}$ Lauren B. SCHMIDT y Erik W. WILLIS, "Systematic investigation of voicing assimilation of Spanish /s/ in Mexico City", Presentación en Laboratory Approacbes to Romance Phonology 2010. Rebeca CAMPOS-ASTORKIZA, "Voicing assimilation as gestural blending: acoustic evidence from Spanish", Presentación en 20th Manchester Phonology Meeting, 2012. John LIPSKI, "/s/ voicing in Ecuadorian Spanish: Patterns and Principles of Consonantal Modification", Lingua, 79, North-Holland, 1989, 49-71.

10 Patrycja STRYCHARCZUK y Ellen SIMON, "Obstruents before sonorants. The case of West-Flemish", Natural Language and Linguistic Theory, en prensa.
} 


\section{Metodologia}

Como ya se ha mencionado, en el presente artículo damos cuenta de un estudio piloto, por lo tanto, se analizarán los datos procedentes de solamente dos personas y no se incluirán cálculos estadísticos. Consideramos necesario el análisis de datos procedentes de aproximadamente 15 personas para poder llegar a conclusiones significativas. Las dos son alumnas de filología hispánica en la Universidad Eötrös Loránd, Budapest, del segundo año del grado (B.A.). Los alumnos para poder seguir sus estudios al final del primer año tienen que pasar un examen de lengua cuyo nivel corresponde aproximadamente al nivel C1 del Marco Europeo de Referencia, asi que los alumnos del segundo año tienen un nivel avanzado.

Nuestras grabaciones están formadas por listas de oraciones leídas en la habitación insonorizada del Laboratorio de Fonética del Instituto de Lingüística de la Academia de Ciencias de Hungría. Las oraciones se presentaron en la pantalla de un ordenador en orden aleatorio con la ayuda del software SpeechRecorder. Cada oración apareció cinco veces pero la primera incidencia se descartó porque se considera que pertenece a la fase de familiarización. Usamos un micrófono Sony ECM-MS907 y una tarjeta de sonido exterior MAudio MobilePre USB preamplifier. Las grabaciones las hicimos en la segunda mitad del curso de fonética donde las alumnas recibieron instrucciones explícitas sobre estos procesos de asimilación, pero todavia no habían tenido tiempo para consolidar sus conocimientos. Nuestros resultados se basan en medidas acústicas del grado de sonoridad.

La sonoridad se midió en base a la periodicidad de la onda sonora, la frecuencia fundamental fo en el espectrograma, la presencia de las líneas de sonoridad en el espectrograma y el informe de Praat. ${ }^{11}$ Usamos los ajustes básicos de Praat: campo de tono $75 \mathrm{~Hz}-500 \mathrm{~Hz}$; factor máximo de periodo: 1,3; factor máximo de amplitud: 1, 6. Los ajustes de tono se optimizaron para el análisis de voz. Otro procedimiento para medir la sonoridad es desde el punto de vista perceptivo, como lo hace Simon (2010). ${ }^{12}$ En el estudio de Simon dos expertos fonéticos clasificaron los segmentos en dos categorías: sordo vs. sonoro a partir de la escucha de los datos. Simon indica que este procedimiento se parece más al habla natural puesto que los hablantes y sus interlocutores hacen lo mismo. El punto débil de este procedimiento es el factor subjetivo, aunque tenemos que admitir que para percibir un segmento como sordo o

"Paul BOERSMA y David WEENIK, Praat: Doing phonetics by computer (versión 4.3.19). Descargado el 20 de julio de 2005. (herp://www.praat.org/)

12 SIMON, op. cit. 
sonoro entran en juego varios correlatos fonéticos (la longitud, la intensidad, etc. del segmento en cuestión y de los segmentos vecinos) y no solamente la fonación misma. Simon grabó conversaciones espontáneas y la calidad de sus grabaciones tampoco permitió el análisis acústico. En el futuro nos gustaría hacer grabaciones de habla espontánea con los alumnos, pero este procedimiento no permite controlar los contextos (no podemos estar seguros de que nuestros sujetos vayan a producir cada tipo de secuencia que nos interesa examinar). Nosotros por esta razón optamos por el habla leída. Consideramos que nuestros sujetos, al tener que leer cada oración cinco veces, estaban en condiciones de producir enunciados bastante cercanos al habla espontánea. Otro factor importante que tuvimos en cuenta para fines instructivos fue hacer grabaciones antes y después del curso de fonética, a fin de poder llegar a conclusiones sobre las posibilidades de aprender este tipo de procesos y los efectos que la lengua materna y la instrucción explícita juegan en la adquisición.

\section{Resultados}

Nuestros resultados confirman que para la adquisición de este tipo de procesos inconscientes se necesita una reflexión explícita profunda y detallada sobre el sistema fonético de la lengua extranjera y muchisima práctica. Nuestras alumnas, en cuanto a los procesos de sonoridad, tienen una pronunciación muy "húngara". En todos los contextos de obstruyente + obstruyente aplican asimilación regresiva: ábside [ps], pub tan $[\mathrm{pt}]$, las gomas $[\mathrm{zg}]$. Tenemos que destacar que tanto las oclusivas sordas como las sonoras se pronuncian como tal, es decir, con un cierre completo y una explosión en todas las posiciones en el 96\% de los casos. Esta falta de pronunciación es muy llamativa y les presta a estas alumnas un acento extranjero bastante marcado. Volviendo a la secuencia de dos obstruyentes, es un caso claro de transferencia de L1 que generalmente - volveremos a la sonorización de la /s/ más adelante- no se puede considerar ni negativa ni positiva puesto que la realización con la asimilación de sonoridad es posible. Si la $\mathrm{C}_{1}$ es oclusiva, la pronunciación oclusiva es posible pero no es la variante preferida por los hablantes nativos, independientemente de la concordancia de sonoridad. Las alumnas húngaras pronuncian acto $[\mathrm{kt}]$ en vez de $[\mathrm{xt}] \circ[\gamma \mathrm{t}]$, como prefieren los nativos. En la expresión el pub de al lado las dos sujetos pronunciaron [bd], lo que es correcto en cuanto a la sonoridad, pero los nativos simplemente omiten la /b/o la secuencia más bien se realiza como $[\beta \delta] .{ }^{13}$

\footnotetext{
${ }^{13} \mathrm{La}$ evaluación estadística de los datos acústicos sobre la asimilación de sonoridad procedentes de hablantes narivos está en progreso.
} 
En cuanto al ensordecimiento a final de palabra, en la palabra ciudad una de las alumnas siempre pronunció [d] lo que podemos considerar incorrecto y es una transferencia negativa del húngaro. La oura realizó el segmento como una interdental sorda o ensordecida. En los préstamos recientes también aparecen otras oclusivas. La palabra pub, por ejemplo, se pronuncia por los españoles con una bilabial sorda sin explosión (o se elide el segmento). Una de nuestras alumnas lo pronunció con una [b] ensordecida que no llegó a ser [p] por la falta de intensidad y duración de la explosión, la cual es también la pronunciación preferida por los nativos. La otra lo pronunció con [b].

El caso de la sonorización de la /s/ en la pronunciación de las dos alumnas corresponde al patrón húngaro, es decir, la $/ \mathrm{s} / \mathrm{se}$ pronuncia sonora delante de una obstruyente sonora (de[z]de, ra[z]go) pero sorda delante de resonantes $i[\mathrm{~s}] l a$. La palabra mismo, que es el ejemplo más frecuente en los libros de gramática, fue pronunciada por una de nuestras alumnas con una sibilante sonora en el $75 \%$ de los casos. Para terminar tenemos que mencionar que una de nuestras alumnas en algunos casos pronunció la /s/ intervocálica en las palabras presente y básico con [z], lo que probablemente se debe a la influencia del inglés. Es una transferencia negativa pero no de L1 sino de L3 que se apoya por la aerodinámica, ya que las vibraciones laringeas invaden por los dos lados la consonante sorda.

\section{Conclusiones}

En este articulo hemos presentado los resultados de un estudio piloto sobre la adquisición del sistema laríngeo del español por parte de dos alumnas húngaras de nivel avanzado. Hemos demostrado que la adquisición de este tipo de procesos subfonémicos e inconscientes requiere mucha práctica y reflexión explícita. En el futuro realizaremos el estudio completo recopilando datos antes y después del curso de fonética, grabaremos hablas espontáneas y frases leídas en laboratorio, haremos medidas acústicas y categorización perceptiva. Planteamos que la adquisición del ensordecimiento final es más fácil que la sonorización por las resonantes puesto que el primero es un proceso tipológicamente no marcado, mientras que el segundo es marcado. 